\title{
Threshold Dynamics of a Stochastic SIR Model with Vertical Transmission and Vaccination
}

\author{
Anqi Miao, ${ }^{1}$ Jian Zhang, ${ }^{1}$ Tongqian Zhang, ${ }^{1,2}$ and B. G. Sampath Aruna Pradeep ${ }^{3}$ \\ ${ }^{1}$ College of Mathematics and Systems Science, Shandong University of Science and Technology, Qingdao 266590, China \\ ${ }^{2}$ State Key Laboratory of Mining Disaster Prevention and Control Co-Founded by Shandong Province and the Ministry of \\ Science and Technology, Shandong University of Science and Technology, Qingdao 266590, China \\ ${ }^{3}$ Department of Mathematics, University of Ruhuna, 81000 Matara, Sri Lanka
}

Correspondence should be addressed to Tongqian Zhang; zhangtongqian@sdust.edu.cn

Received 25 March 2017; Accepted 4 June 2017; Published 6 July 2017

Academic Editor: Gul Zaman

Copyright (C) 2017 Anqi Miao et al. This is an open access article distributed under the Creative Commons Attribution License, which permits unrestricted use, distribution, and reproduction in any medium, provided the original work is properly cited.

A stochastic SIR model with vertical transmission and vaccination is proposed and investigated in this paper. The threshold dynamics are explored when the noise is small. The conditions for the extinction or persistence of infectious diseases are deduced. Our results show that large noise can lead to the extinction of infectious diseases which is conducive to epidemic diseases control.

\section{Introduction}

The history of mankind is filled with struggle with diseases. Infectious diseases such as smallpox, cholera, plague of leprosy, diphtheria, syphilis, typhus fever, malaria, rabies, and tuberculosis have threatened the health of human beings. People have realized the importance of quantitative studies on the spread of infectious diseases to predict and to control them. It can be known from referring to the literature [1-4] that, with the aid of the establishment of infectious disease models, people can understand the crucial laws of infectious diseases and provide reliable and enough information to predict and control infectious diseases. For example, as early as 1760, Bernoulli and Blower [5] proposed the first mathematical model in epidemiology for studying the spread and inoculation of smallpox. Further, in 1927, Kermack and McKendrick [6] proposed the concept of the so-called "compartmental model," in which all the population was classified into three compartments: susceptible compartment $S$, infected compartment $I$, and removed compartment $R$. It is assumed in the model that the susceptible class can transform into the infective class through contact with infected individuals, and the infectives can recover through treatment so that they have permanent immunity. Therefore, it is now well known that many scholars have paid attention to SIR models; as a result, it can be seen in the literature that a large number of mathematical models of ordinary differential equations, delay differential equations, and partial differential equations have been constructed to study the spread of infectious diseases (see, e.g., [7-23]). In the last decades, we observed that scholars published few papers in scientific journals related to mathematics considering infectious diseases with vertical transmission which are transmitted from parents to their offspring (e.g., [1,24-26]). Although scholars neglect the effect of vertical transmission, it is very important to study the real situation of the transmission of infectious diseases. The current diseases affecting humanity such as AIDS [27-31], Chagas' disease [32-34], hepatitis B [35, 36], and hepatitis C [37] are vertically transmitted. From this, it can be clearly seen that mathematical modeling including vertical transmission, horizontal transmission, and vaccination $[38,39]$ is more realistic than without them. Therefore, in this study, we have focused our attention on this and an SIR epidemic model involving vertical transmission and vaccination was proposed as follows [1,24] (see Figure 1):

$$
\begin{aligned}
\dot{S}(t)= & -\beta S(t) I(t)+(1-m) b(S(t)+R(t)) \\
& +p b^{\prime} I(t)-b S(t), \\
\dot{I}(t)= & \beta S(t) I(t)+q b^{\prime} I(t)-b^{\prime} I(t)-\gamma I(t), \\
\dot{R}(t)= & \gamma I(t)-b R(t)+m b(S(t)+R(t)),
\end{aligned}
$$




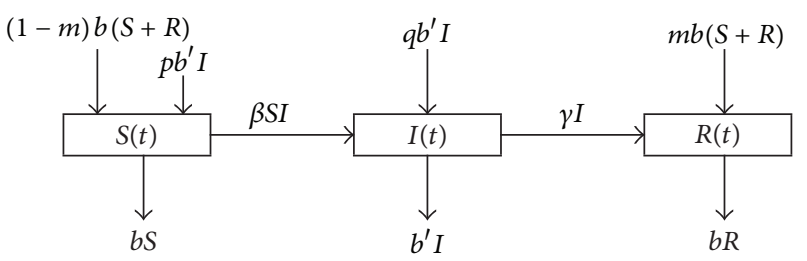

FIGURE 1: The compartmental diagram for the SIR model with vertical transmission and vaccination.

where $S(t), I(t)$, and $R(t)$ represent the members of the susceptible, the infectious, and the removed or the recovered members from infection, respectively. $b$ is the birth and death rate of $S(t)$ and $R(t), b^{\prime}$ is the birth and death rate of $I(t), \beta$ is the contact rate, and $m(0<m<1)$ is the vaccination proportion to the newborn from $S(t)$ and $R(t)$. Then, constant $p(0<p<1)$ is the proportion of the offspring of infective parents that are susceptible individuals and $p+q=1 . \gamma$ is the recovery rate of the infective individuals. Obviously, the total population size is normalized to one, and the basic reproductive number of system (1) is $R_{0}=\beta(1-m) /\left(p b^{\prime}+\gamma\right)$. By constructing a Lyapunov function and using the LaSalle invariance principle, we can show that if $R_{0}<1$, the infectionfree equilibrium $P_{0}(1-m, 0, m)$ is globally asymptotically stable, while if $R_{0}>1$, the infection-free equilibrium $P_{0}$ is unstable and the endemic equilibrium $P^{*}\left(S^{*}, I^{*}, R^{*}\right)$ is globally asymptotically stable.

In fact, the spread of diseases is inevitably disturbed by the influence of random factors; the stochastic epidemic system is more in line with the actual situation. Therefore, epidemic systems described by stochastic differential equations have been paid extensive attention in recent years (see, e.g., [40-46]). Various stochastic perturbation approaches have been introduced into epidemic systems and excellent results have been obtained. In this study, our main objective is to introduce four approaches. The first one is to analyze epidemic systems including the environment noise by using the method of time Markov chain (see, e.g., [47-51]). The second one is to consider the parameters' perturbation (see, e.g., [52-72]). The third one is to introduce Lévy jump noise into the system [73-75]. The fourth one is to investigate stochastic perturbation around the positive equilibria of deterministic systems (see, e.g., [41, 42, 76-78]).

Parameter perturbation induced by white noises is an important and common form to describe the effect of stochasticity. In this paper, we adopt the perturbation with white noises, that is, $\beta \rightarrow \beta+\sigma \dot{B}(t)$, where $B(t)$ is a standard Brownian motion with intensity $\sigma^{2}>0$. Then, the resultant system transforms into the following form:

$$
\begin{aligned}
& d S(t)=(-\beta S(t) I(t)+(1-m) b(S(t)+R(t)) \\
& \left.\quad+p b^{\prime} I(t)-b S(t)\right) d t-\sigma S(t) I(t) d B(t), \\
& d I(t)=\left(\beta S(t) I(t)+q b^{\prime} I(t)-b^{\prime} I(t)-\gamma I(t)\right) d t \\
& \quad+\sigma S(t) I(t) d B(t), \\
& d R(t)=(\gamma I(t)-b R(t)+m b(S(t)+R(t))) d t .
\end{aligned}
$$

This paper is organized as follows. In Section 3, we will discuss the extinction of infectious diseases and explore the conditions leading to the extinction of infectious diseases. In Section 4, we will deduce the condition for a disease in order to be persistent.

\section{Preliminaries}

Throughout this paper, we let $\mathbb{R}^{d}$ : be the $d$-dimensional Euclidean space. $\mathbb{R}_{+}^{d}:=\left\{x \in \mathbb{R}^{d}: x_{i}>0,1 \leq i \leq d\right\}$, that is, the positive cone.

Let $\left\{B_{t}\right\}_{t \geq 0}$ be a one-dimensional Brownian motion defined on the complete probability space $(\Omega, \mathscr{F}, \mathscr{P})$ adapted to the filtration $\{\mathscr{F}\}_{t \geq 0}$. Let $\mathscr{L}^{1}\left(\mathbb{R}_{+} ; \mathbb{R}^{d}\right)$ denote the family of all $\mathbb{R}^{d}$-valued measurable $\left\{\mathscr{F}_{t}\right\}$-adapted processes $f=$ $\{f(t)\}_{t \geq 0}$ such that

$$
\int_{0}^{T}|f(t)| d t<\infty \quad \text { a.s. for every } T>0 .
$$

Let $C^{2,1}\left(\mathbb{R}^{d} \times \mathbb{R}_{+} ; \mathbb{R}\right)$ denote the family of all real-valued functions $V(x, t)$ defined on $\mathbb{R}^{d} \times \mathbb{R}_{+}$such that they are continuously twice differentiable in $x$ and once in $t$. We set

$$
\begin{aligned}
V_{t} & =\frac{\partial V}{\partial t} \\
V_{x} & =\left(\frac{\partial V}{\partial x_{1}}, \frac{\partial V}{\partial x_{2}}, \ldots, \frac{\partial V}{\partial x_{d}}\right) \\
V_{x x} & =\left(\frac{\partial^{2} V}{\partial x_{i} \partial x_{j}}\right)_{d \times d}=\left(\begin{array}{ccc}
\frac{\partial^{2} V}{\partial x_{1} \partial x_{1}} & \cdots & \frac{\partial^{2} V}{\partial x_{1} \partial x_{d}} \\
\vdots & & \vdots \\
\frac{\partial^{2} V}{\partial x_{d} \partial x_{1}} & \cdots & \frac{\partial^{2} V}{\partial x_{d} \partial x_{d}}
\end{array}\right) .
\end{aligned}
$$

Clearly, when $V \in C^{2,1}\left(R \times R_{+} ; R\right)$, we have $V_{x}=\partial V / \partial x$, $V_{x x}=\partial^{2} V / \partial x^{2}$. Then, we have the following.

Lemma 1 (one-dimensional Itô's formula $[40,79,80]$ ). Let $x(t)$ be an Itô process on $t \geq 0$ with the stochastic differential

$$
d x(t)=f(t) d t+g(t) d B_{t},
$$

where $f \in \mathscr{L}^{1}\left(\mathbb{R}_{+} ; \mathbb{R}\right)$ and $g \in \mathscr{L}^{2}\left(\mathbb{R}_{+} ; \mathbb{R}\right)$. Let $V \in$ $C^{2,1}\left(\mathbb{R}^{d} \times \mathbb{R}_{+} ; \mathbb{R}\right)$. Then, $V(x(t), t)$ is again an Itô process with the stochastic differential given by

$$
\begin{aligned}
& d V(x(t), t)=\left[V_{t}(x(t), t)+V_{x}(x(t), t) f(t)\right. \\
& \left.+\frac{1}{2} V_{x x}(x(t), t) g^{2}(t)\right] d t+V_{x}(x(t), t) \\
& \cdot g(t) d B_{t},
\end{aligned}
$$

almost surely.

By using the methods from Lahrouz and Omari [81], we can prove the following lemma. 
Lemma 2. For any initial value $(S(0), I(0), R(0)) \in R_{+}^{3}$, there exists a unique solution $(S(t), I(t), R(t))$ to system (2) on $t \geq 0$, and the solution will remain in $R_{+}^{3}$ with probability one, namely.

Lemma 3. On the basis of Lemma 2, if $S(0)+I(0)+R(0) \leq 1$, then $S(t)+I(t)+R(t) \leq 1$, almost surely. Thus, the region $\Gamma=\left\{(S, I, R) \in R_{+}^{3}: S>0, I \geq 0, R>0, S+I+R \leq 1\right\}$ is a positively invariant set of system (2).

\section{Extinction}

In this section, we deduce the condition which will cause a disease to die out.

Definition 4. For system (2), the infected individual $I(t)$ is said to be extinctive if $\lim _{t \rightarrow+\infty} I(t)=0$, almost surely.

Let us introduce

$$
R^{*}=\frac{R_{0}}{1-m}-\frac{\sigma^{2}}{2\left(p b^{\prime}+\gamma\right)}
$$

for convenience; then, we have the following results that we have mentioned in the following theorem.

Theorem 5. If $\sigma^{2}>\max \left\{\beta, \beta^{2} / 2\left(p b^{\prime}+\gamma\right)\right\}$ or $\sigma^{2}<\beta$ and $R^{*}<$ 1 , then the infected individual of system (2) goes to extinction almost surely.

Proof. Let $(S(t), I(t), R(t))$ be a solution of system (2) with initial value $(S(0), I(0), R(0)) \in R_{+}^{3}$. Applying Itô’s formula to the second equation of system (2) leads to

$$
\begin{aligned}
\mathrm{d} \ln I(t)= & \left(\beta S(t)-\left(p b^{\prime}+\gamma\right)-\frac{\sigma^{2}}{2} S^{2}(t)\right) \mathrm{d} t \\
& +\sigma S(t) \mathrm{d} B(t) .
\end{aligned}
$$

Integrating both sides of (8) from 0 to $t$ gives

$$
\begin{aligned}
\ln I(t)= & \int_{0}^{t}\left(\beta S(\tau)-\frac{\sigma^{2}}{2} S^{2}(\tau)\right) \mathrm{d} \tau-\left(p b^{\prime}+\gamma\right) t \\
& +M(t)+\ln I(0),
\end{aligned}
$$

where $M(t)=\int_{0}^{t} \sigma S(\tau) \mathrm{d} B(\tau)$ and $M(t)$ is the local continuous martingale with $M(0)=0$. Next, we have two cases to be discussed, depending on whether $\sigma^{2}>\beta$.

If $\sigma^{2}>\beta$, we can easily see from (9) that

$$
\ln I(t) \leq\left(\frac{\beta^{2}}{2 \sigma^{2}}-\left(p b^{\prime}+\gamma\right)\right) t+M(t)+\ln I(0) .
$$

Dividing both sides of (10) by $t>0$, we have

$$
\frac{\ln I(t)}{t} \leq-\left(p b^{\prime}+\gamma-\frac{\beta^{2}}{2 \sigma^{2}}\right)+\frac{M(t)}{t}+\frac{\ln I(0)}{t} .
$$

Since $\lim \sup _{t \rightarrow \infty}\left(\langle M(t), M(t)\rangle_{t} / t\right)<\sigma^{2}<\infty$ almost surely, by the large number theorem for martingales (see, e.g., [53]), one can obtain that

$$
\lim _{t \rightarrow+\infty} \frac{M(t)}{t}=0 .
$$

Then, taking the limit superior on both sides of (11) leads to

$$
\limsup _{t \rightarrow+\infty} \frac{\ln I(t)}{t} \leq-\left(p b^{\prime}+\gamma-\frac{\beta^{2}}{2 \sigma^{2}}\right)<0,
$$

when $\sigma^{2}>\beta^{2} / 2\left(p b^{\prime}+\gamma\right)$, which implies $\lim _{t \rightarrow+\infty} I(t)=0$.

If $\sigma^{2}<\beta$, similarly, one can have that

$$
\ln I(t) \leq\left(\beta-\left(p b^{\prime}+\gamma\right)-\frac{\sigma^{2}}{2}\right) t+M(t)+\ln I(0) .
$$

Dividing both sides of (14) by $t>0$, we have

$$
\begin{aligned}
\frac{\ln I(t)}{t} \leq & \left(p b^{\prime}+\gamma\right)\left[\frac{\beta}{p b^{\prime}+\gamma}-\frac{\sigma^{2}}{2\left(p b^{\prime}+\gamma\right)}-1\right] \\
& +\frac{M(t)}{t}+\frac{\ln I(0)}{t} .
\end{aligned}
$$

By taking the superior limit on both sides of (15), one can have that

$$
\limsup _{t \rightarrow+\infty} \frac{\ln I(t)}{t} \leq\left(p b^{\prime}+\gamma\right)\left(R^{*}-1\right) .
$$

Then, when $R^{*}<1$, we obtain

$$
\limsup _{t \rightarrow+\infty} \frac{\ln I(t)}{t}<0
$$

which implies $\lim _{t \rightarrow+\infty} I(t)=0$. This completes the proof of Theorem 5 .

Remark 6. Theorem 5 shows that when $\sigma^{2}>\max \left\{\beta, \beta^{2} /\right.$ $\left.2\left(p b^{\prime}+\gamma\right)\right\}$, the infectious disease of system (2) goes to extinction almost surely; namely, large white noise stochastic disturbance is conducive to control infectious diseases. When the white noise is not large and $R^{*}<1$, the infectious disease of system (2) also goes to extinction almost surely; then, $R^{*}$ is the threshold associated with the extinction of infectious diseases.

\section{Persistence in Mean}

Definition 7. For system (2), the infected individual $I(t)$ is said to be permanent in mean if $\liminf _{t \rightarrow+\infty}\langle I(t)\rangle>0$, almost surely, where $\langle I(t)\rangle$ is defined as $(1 / t) \int_{0}^{t} I(\tau) \mathrm{d} \tau$.

Let us denote

$$
\mathscr{R}^{* *}=R_{0}-\frac{\sigma^{2}}{2\left(p b^{\prime}+\gamma\right)}
$$

for convenience; then, we have the following results that we have mentioned in the following theorem. 


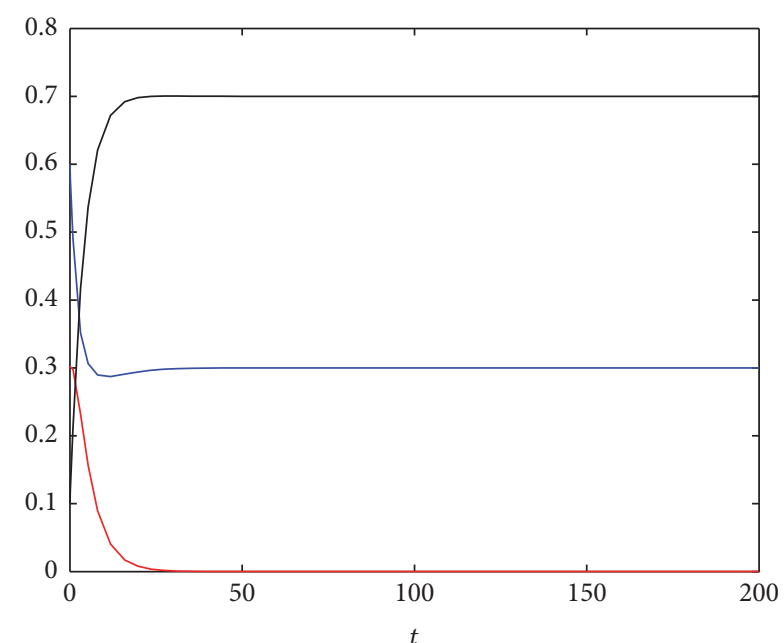

$S(t)$ of deterministic system

$(t)$ of deterministic system

$R(t)$ of deterministic system

(a) Time series for $S(t), I(t), R(t)$ where $m=0.7$ and $R_{0}=0.5455$

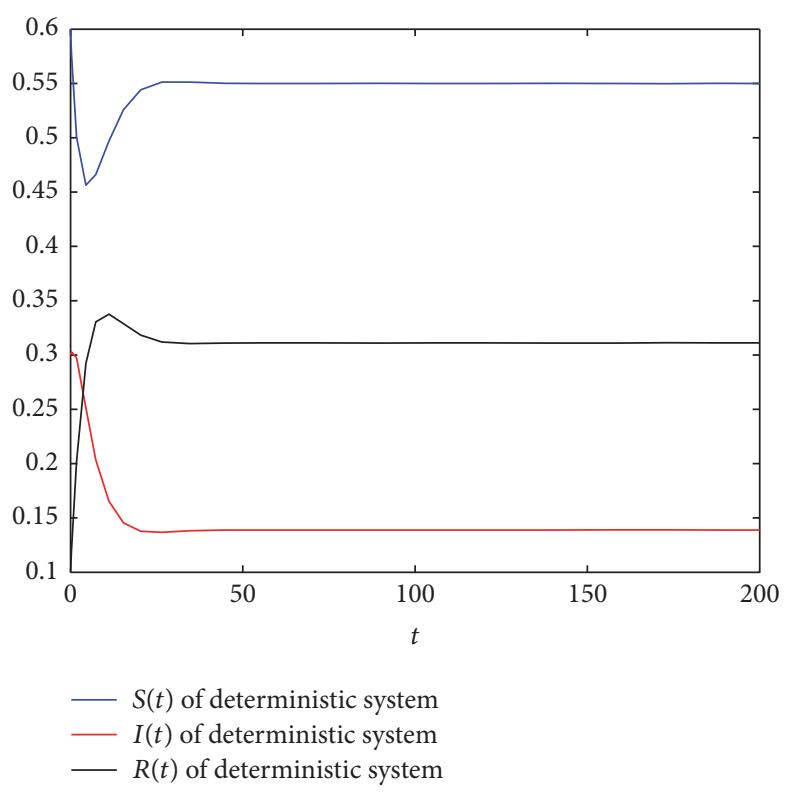

(b) Time series for $S(t), I(t), R(t)$ where $m=0.2$ and $R_{0}=1.4545$

FIGURE 2: Illustration for the deterministic SIR system where $\beta=0.8, p=0.6, b=0.2, b^{\prime}=0.4$, and $\gamma=0.2$.

Theorem 8. If $\mathscr{R}^{* *}>1$, then the infected individual $I(t)$ is persistent in mean; moreover, $I(t)$ satisfies

$$
\liminf _{t \rightarrow+\infty}\langle I(t)\rangle \geq \frac{\left(p b^{\prime}+\gamma\right)}{\beta(1-m+\gamma / b)}\left(R^{* *}-1\right)
$$

almost surely.

Proof. Integrating from 0 to $t$ and dividing by $t(t>0)$ on both sides of the third equation of system (2) yield

$$
\begin{aligned}
\frac{R(t)-R(0)}{t}= & \gamma\langle I(t)\rangle+m b\langle S(t)\rangle \\
& -(1-m) b\langle R(t)\rangle \triangleq \Theta(t) .
\end{aligned}
$$

Note that $\langle S(t)\rangle+\langle I(t)\rangle+\langle R(t)\rangle=1$; then, one can get

$$
\langle S(t)\rangle=(1-m)+\frac{\Theta(t)}{b}-\left(1-m+\frac{\gamma}{b}\right)\langle I(t)\rangle .
$$

Applying Itô's formula gives

$$
\begin{aligned}
\mathrm{d}(\ln I(t))= & {\left[\beta S(t)-\left(p b^{\prime}+\gamma\right)-\frac{\sigma^{2}}{2} S^{2}(t)\right] \mathrm{d} t } \\
& +\sigma S(t) \mathrm{d} B(t) \\
\geq & {\left[\beta S(t)-\left(p b^{\prime}+\gamma\right)-\frac{\sigma^{2}}{2}\right] \mathrm{d} t } \\
& +\sigma S(t) \mathrm{d} B(t) .
\end{aligned}
$$

Integrating from 0 to $t$ and dividing by $t(t>0)$ on both sides of (22) yield

$$
\begin{aligned}
& \frac{\ln I(t)-\ln I(0)}{t} \\
& \geq \beta\langle S(t)\rangle-\left[\left(p b^{\prime}+\gamma\right)+\frac{\sigma^{2}}{2}\right]+\frac{M(t)}{t} \\
& =\beta\left(1-m+\frac{\Theta(t)}{b}-\left(1-m+\frac{\gamma}{b}\right)\langle I(t)\rangle\right) \\
& \quad-\left(p b^{\prime}+\gamma+\frac{\sigma^{2}}{2}\right)+\frac{M(t)}{t} .
\end{aligned}
$$

From (23), we obtain

$$
\begin{aligned}
& \langle I(t)\rangle \geq \frac{1}{\beta(1-m+\gamma / b)}\left[\beta(1-m)-\left(p b^{\prime}+\gamma\right)\right. \\
& \left.-\frac{\sigma^{2}}{2}\right]+\frac{1}{\beta(1-m+\gamma / b)}\left[\frac{\beta \Theta(t)}{b}\right. \\
& \left.-\frac{\ln I(t)-\ln I(0)}{t}+\frac{M(t)}{t}\right] .
\end{aligned}
$$

Since both $I(t) \leq 1$ and $R(t) \leq 1$, then one has $\lim _{t \rightarrow+\infty}(R(t) /$ $t)=0, \lim _{t \rightarrow+\infty}(\ln I(t) / t)=0$, and $\lim _{t \rightarrow+\infty} \Theta(t)=0$. Note 


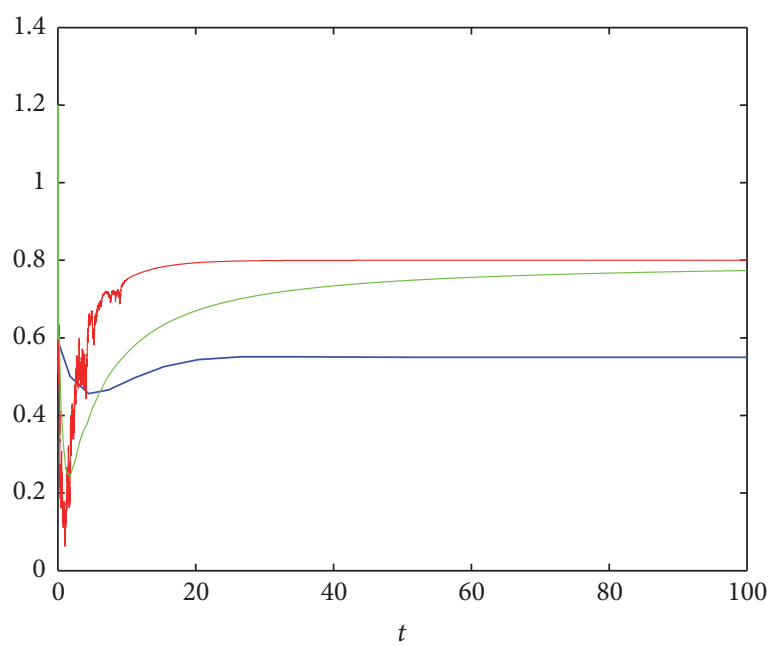

$S(t)$ of system (1)

- $S(t)$ of system (2) $t^{-1} \int_{0}^{t} S(s) \mathrm{d} s$

(a) Time series for $S(t)$ and $\langle S(t)\rangle$

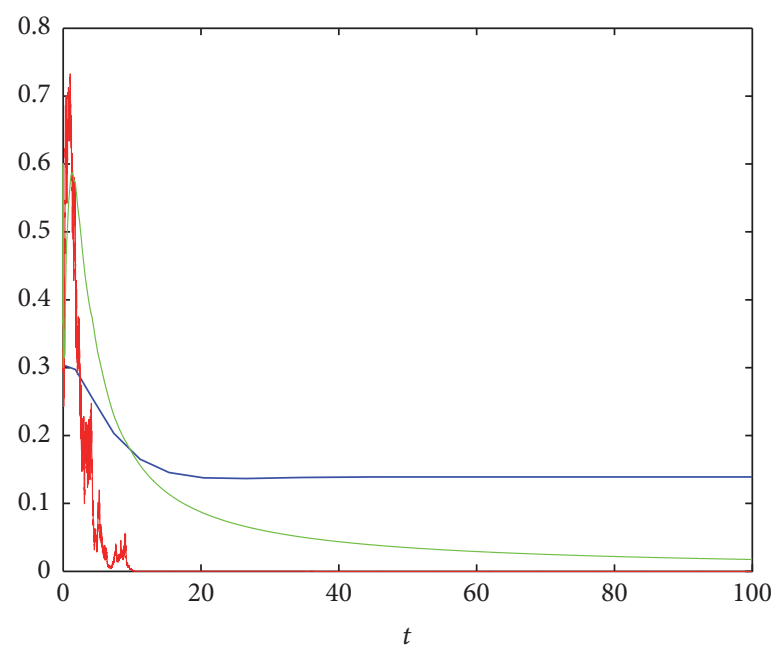

$I(t)$ of system (1)

$I(t)$ of system (2)

$t^{-1} \int_{0}^{t} I(s) \mathrm{d} s$

(b) Time series for $I(t)$ and $\langle I(t)\rangle$

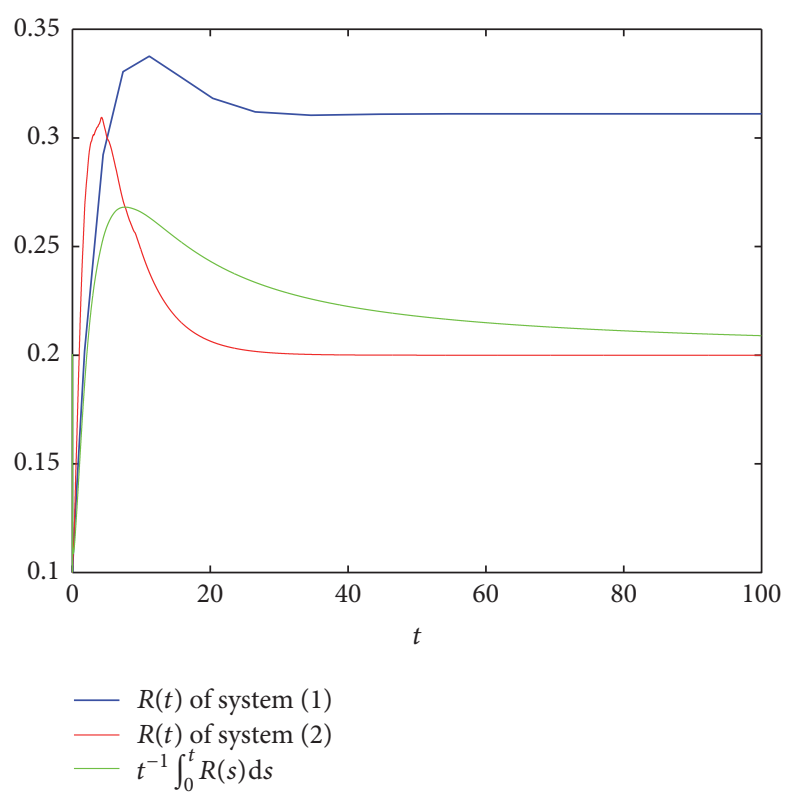

(c) Time series for $R(t)$ and $\langle R(t)\rangle$

FIGURE 3: Comparison of the deterministic system and stochastic system, where $m=0.2, \beta=0.8, p=0.6, b=0.2, b^{\prime}=0.4, \gamma=0.2, \sigma=0.9$, and $R_{0}=1.4545>1$.

that $\lim _{t \rightarrow+\infty}(M(t) / t)=0$; by taking the inferior limit of both sides of (24), we have

$$
\begin{aligned}
& \liminf _{t \rightarrow+\infty}\langle I(t)\rangle \\
& \geq \frac{1}{\beta(1-m+\gamma / b)}\left[\beta(1-m)-p b^{\prime}-\gamma-\frac{\sigma^{2}}{2}\right] \\
& \quad=\frac{\left(p b^{\prime}+\gamma\right)}{\beta(1-m+\gamma / b)}\left(R^{* *}-1\right) .
\end{aligned}
$$

This completes the proof of Theorem 8 .
Remark 9. Theorems 5 and 8 show that the condition for the disease to die out or persist depends on the intensity of white noise disturbances strongly. And small white noise disturbances will be beneficial for long-term prevalence of the disease; conversely, large white noise disturbances may cause the epidemic disease to die out.

\section{Conclusion and Numerical Simulation}

In this paper, a stochastic SIR system with vertical transmission and vaccination is proposed. The threshold dynamics 


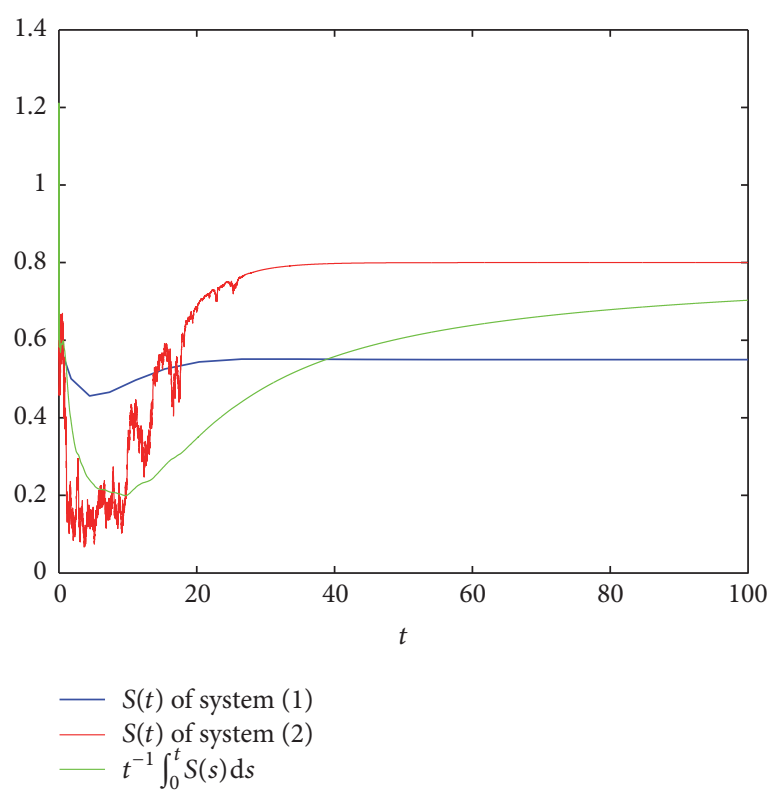

(a) Time series for $S(t)$ and $\langle S(t)\rangle$

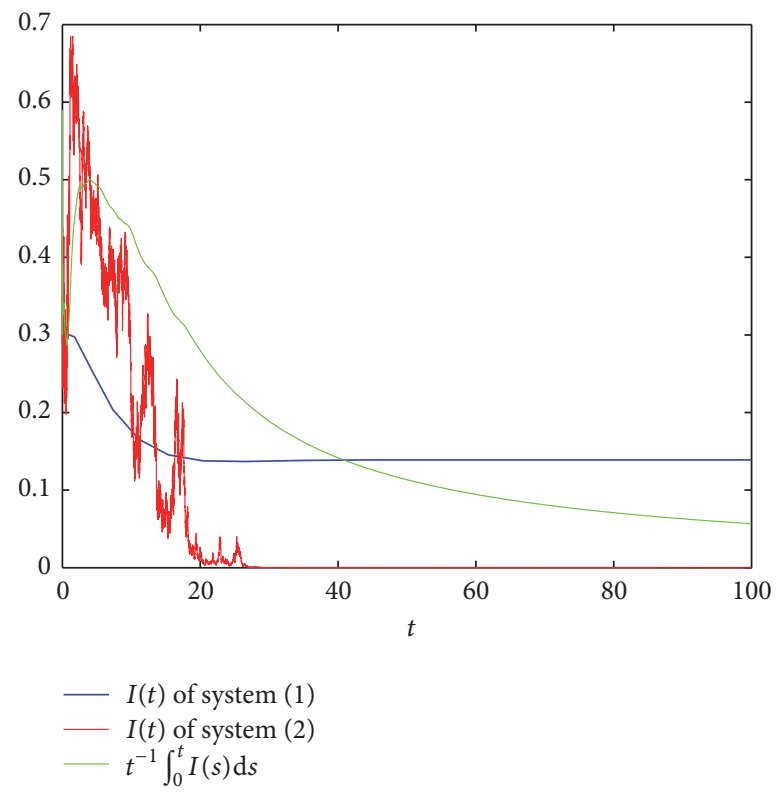

(b) Time series for $I(t)$ and $\langle I(t)\rangle$

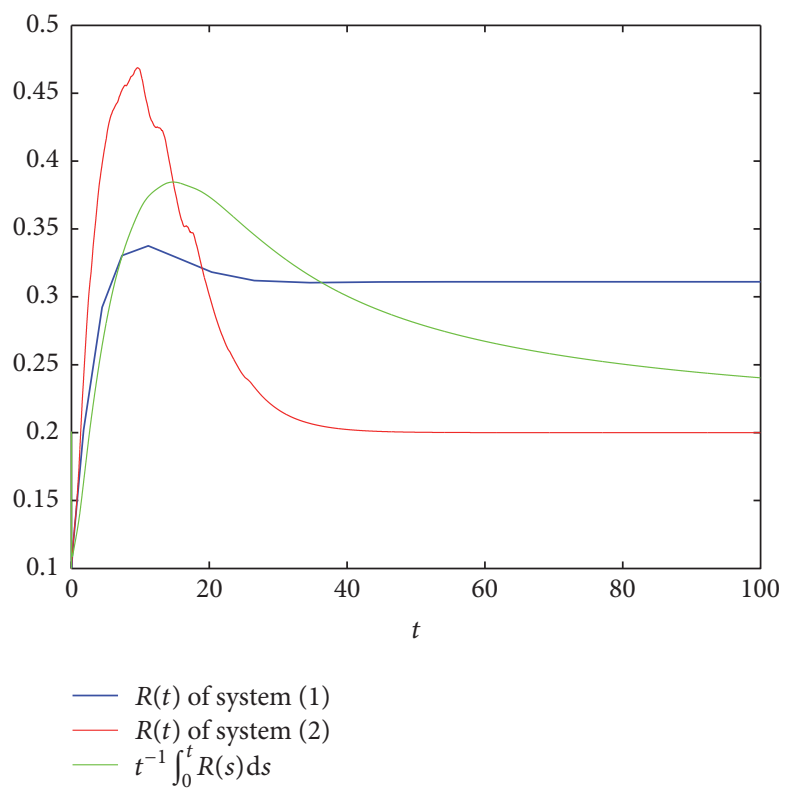

(c) Time series for $R(t)$ and $\langle R(t)\rangle$

FiguRE 4: Comparison of the deterministic system and stochastic system, where $m=0.2, \beta=0.8, p=0.6, b=0.2, b^{\prime}=0.4, \gamma=0.2, \sigma=0.85$, $R^{*}=0.9972$, and $R_{0}=1.4545>1$.

depending on the stochastic perturbation are deduced by using the theory of stochastic differential equation and inequality technique. Our results show that the dynamics of the stochastic system are different with the deterministic case due to the effect of stochastic perturbation, and the persistent diseases in the deterministic system may be eliminated under the stochastic perturbation.

In the following, by employing the Euler Maruyama (EM) method [40], we perform some numerical simulations to illustrate the extinction and persistence of the diseases in the stochastic system and corresponding deterministic system for comparison.

For numerical simulations, we set parameters as $m=0.7$, $\beta=0.8, p=0.6, b=0.2, b^{\prime}=0.4$, and $\gamma=0.2$ in system (1). A simple computation shows that $R_{0}=0.5455<1$, and then system (1) has a stable infection-free equilibrium $P_{0}(0.3,0,0.7)$, which implies that the disease of system (1) will be eliminated ultimately (see Figure 2(a)). If we change $m=$ 0.7 to $m=0.2$, in this case, $R_{0}=1.4545>1$, and then system (1) has a stable infection equilibrium $P^{*}(0.55,0.3111,0.1389)$, 

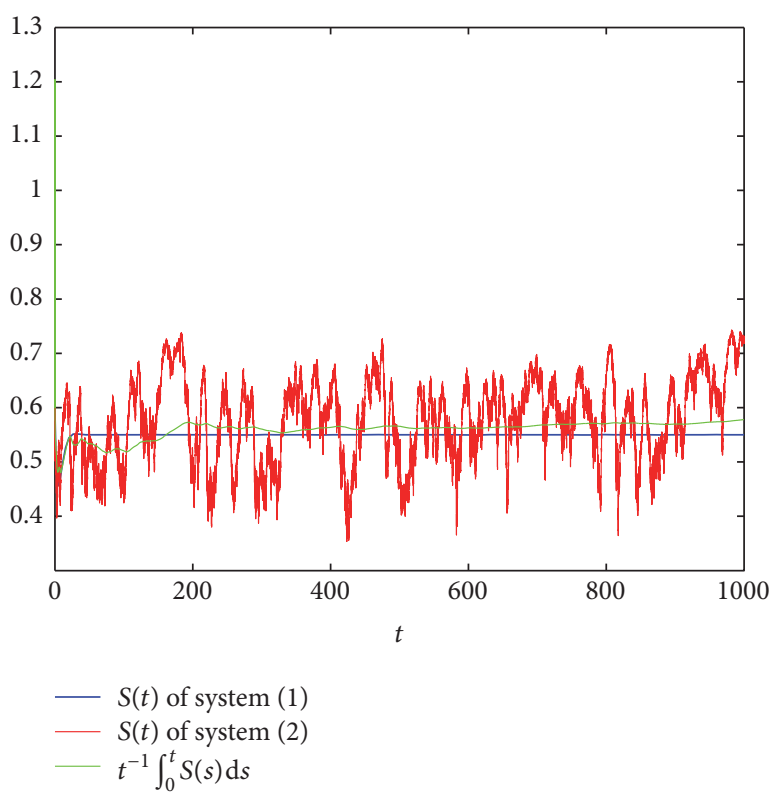

(a) Time series for $S(t)$ and $\langle S(t)\rangle$
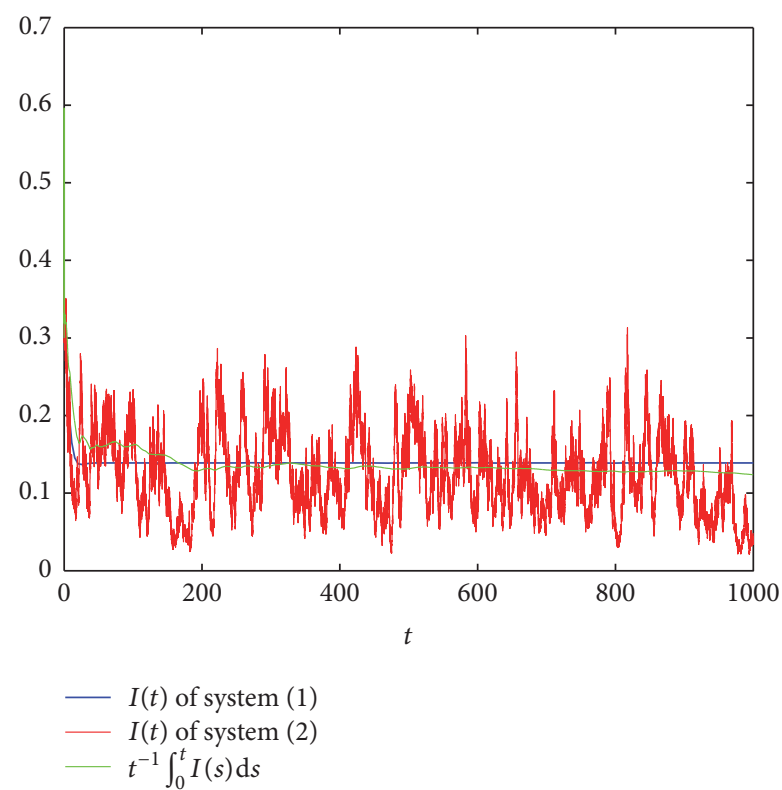

(b) Time series for $I(t)$ and $\langle I(t)\rangle$

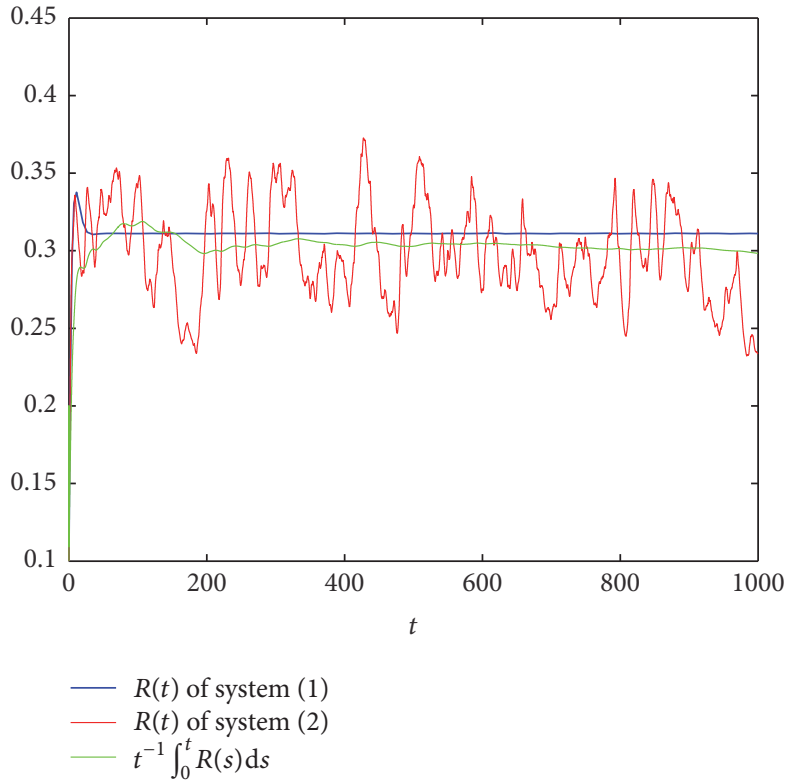

(c) Time series for $R(t)$ and $\langle R(t)\rangle$

Figure 5: Comparison of the deterministic system and stochastic system, where $m=0.2, \beta=0.8, p=0.6, b=0.2, b^{\prime}=0.4, \gamma=0.2, \sigma=0.2$, $R^{* *}=1.4091$, and $R_{0}=1.4545>1$.

which implies that the disease of system (1) will be persistent ultimately (see Figure 2(b)).

Next, we consider the effect of stochastic white noise based on the persistent system. Let $\sigma=0.9$, and obviously, $\sigma^{2}>\max \left\{\beta, \beta^{2} / 2\left(p b^{\prime}+\gamma\right)\right\}$; by Theorem 5 , the disease dies out under a large white noise disturbance (see Figure 3 ). If we change $\sigma$ to 0.85 , in this case, $\sigma^{2}<\beta^{2} / 2\left(p b^{\prime}+\gamma\right)$ and $R^{*}=0.9972=<1$; then, by Theorem 5 , the disease dies out (see Figure 4). If we reduce the intensity of noise $\sigma$ to 0.2 , obviously, $R^{* *}=1.4091>1$; by Theorem 8 , the disease is persistent (see Figure 5).

\section{Conflicts of Interest}

The authors declare that there are no conflicts of interest regarding the publication of this paper.

\section{Acknowledgments}

This work is supported by Shandong Provincial Natural Science Foundation of China (no. ZR2015AQ001), the National Natural Science Foundation of China (no. 11371230), and Research Funds for Joint Innovative Center for Safe and Effective Mining Technology and Equipment 
of Coal Resources by Shandong Province and SDUST (2014TDJH102).

\section{References}

[1] R. Anderson and R. May, Infectious Diseases in Humans: Dynamics and Control, Oxford University Press, Oxford, UK, 1992.

[2] C. V. De-León, "On the global stability of SIS, SIR and SIRS epidemic models with standard incidence," Chaos, Solitons \& Fractals, vol. 44, no. 12, pp. 1106-1110, 2011.

[3] F. Brauer, Epidemic Models in Populations of Varying Size, Springer, Berlin, Germany, 1989.

[4] H. W. Hethcote, "The mathematics of infectious diseases," SIAM Review, vol. 42, no. 4, pp. 599-653, 2000.

[5] D. Bernoulli and S. Blower, "An attempt at a new analysis of the mortality caused by smallpox and of the advantages of inoculation to prevent it," Reviews in Medical Virology, vol. 14, no. 5, pp. 275-288, 2004.

[6] W. O. Kermack and A. G. McKendrick, "A contribution to the mathematical theory of epidemics," Proceedings of the Royal Society of London A: Mathematical, Physical and Engineering Sciences, vol. 115, no. 772, pp. 700-721, 1927.

[7] G. Huang and Y. Takeuchi, "Global analysis on delay epidemiological dynamic models with nonlinear incidence," Journal of Mathematical Biology, vol. 63, no. 1, pp. 125-139, 2011.

[8] G. Huang, Y. Takeuchi, W. Ma, and D. Wei, "Global stability for delay SIR and SEIR epidemic models with nonlinear incidence rate," Bulletin of Mathematical Biology, vol. 72, no. 5, pp. 11921207, 2010.

[9] Z. Hu, W. Ma, and S. Ruan, "Analysis of SIR epidemic models with nonlinear incidence rate and treatment," Mathematical Biosciences, vol. 238, no. 1, pp. 12-20, 2012.

[10] B. G. Pradeep and W. Ma, "Global stability of a delayed mosquito-transmitted disease model with stage structure," Electronic Journal of Differential Equations, vol. 10, pp. 1-19, 2015.

[11] Z. Jiang and W. Ma, "Permanence of a delayed SIR epidemic model with general nonlinear incidence rate," Mathematical Methods in the Applied Sciences, vol. 38, no. 3, pp. 505-516, 2015.

[12] T. Zhang, X. Meng, T. Zhang, and Y. Song, "Global dynamics for a new high-dimensional SIR model with distributed delay," Applied Mathematics and Computation, vol. 218, no. 24, pp. 11806-11819, 2012.

[13] W. Zhao, T. Zhang, Z. Chang, X. Meng, and Y. Liu, "Dynamical analysis of SIR epidemic models with distributed delay," Journal of Applied Mathematics, vol. 2013, Article ID 154387, 15 pages, 2013.

[14] K. I. Kim, Z. Lin, and L. Zhang, "Avian-human influenza epidemic model with diffusion," Nonlinear Analysis: Real World Applications, vol. 11, no. 1, pp. 313-322, 2010.

[15] G. Zaman, Y. Han Kang, and I. H. Jung, "Stability analysis and optimal vaccination of an SIR epidemic model," BioSystems, vol. 93, no. 3, pp. 240-249, 2008.

[16] L. Zhang, Z.-C. Wang, and X.-Q. Zhao, "Threshold dynamics of a time periodic reaction-diffusion epidemic model with latent period," Journal of Differential Equations, vol. 258, no. 9, pp. 3011-3036, 2015.

[17] W. Wang and X.-Q. Zhao, "An epidemic model with population dispersal and infection period," SIAM Journal on Applied Mathematics, vol. 66, no. 4, pp. 1454-1472, 2006.
[18] W. Wang, W. Ma, and X. Lai, "Repulsion effect on superinfecting virions by infected cells for virus infection dynamic model with absorption effect and chemotaxis," Nonlinear Analysis: Real World Applications, vol. 33, pp. 253-283, 2017.

[19] W. Wang, W. Ma, and X. Lai, "A diffusive virus infection dynamic model with nonlinear functional response, absorption effect and chemotaxis," Communications in Nonlinear Science and Numerical Simulation, vol. 42, pp. 585-606, 2017.

[20] G. Zaman, Y. H. Kang, and I. H. Jung, "Optimal treatment of an SIR epidemic model with time delay," BioSystems, vol. 98, no. 1, pp. 43-50, 2009.

[21] X. Meng, Y. Yang, and S. Zhao, "Adaptive evolution of virulencerelated traits in a susceptible-infected model with treatment," Abstract and Applied Analysis, vol. 2014, Article ID 891401, 10 pages, 2014.

[22] W. Zhao, J. Li, and X. Meng, "Dynamical analysis of SIR epidemic model with nonlinear pulse vaccination and lifelong immunity," Discrete Dynamics in Nature and Society, vol. 2015, Article ID 848623, 10 pages, 2015.

[23] S. Guo and W. Ma, "Global behavior of delay differential equations model of HIV infection with apoptosis," Discrete and Continuous Dynamical Systems. Series B, vol. 21, no. 1, pp. 103119, 2016.

[24] X. Meng and L. Chen, "The dynamics of a new SIR epidemic model concerning pulse vaccination strategy," Applied Mathematics and Computation, vol. 197, no. 2, pp. 582-597, 2008.

[25] Z. Lu, X. Chi, and L. Chen, "The effect of constant and pulse vaccination on SIR epidemic model with horizontal and vertical transmission," Mathematical and Computer Modelling, vol. 36, no. 9-10, pp. 1039-1057, 2002.

[26] Y. Chen, J. Evans, and M. Feldlaufer, "Horizontal and vertical transmission of viruses in the honey bee, Apis mellifera," Journal of Invertebrate Pathology, vol. 92, no. 3, pp. 152-159, 2006.

[27] S. Sprecher, G. Soumenkoff, F. Puissant, and M. Degueldre, "Vertical transmission of hiv in 15-week fetus," The Lancet, vol. 328, no. 8501, pp. 288-289, 1986.

[28] A. B. Van't Wout, N. A. Kootstra, G. A. Mulder-Kampinga et al., "Macrophage-tropic variants initiate human immunodeficiency virus type 1 infection after sexual, parenteral, and vertical transmission," Journal of Clinical Investigation, vol. 94, no. 5, pp. 2060-2067, 1994.

[29] T. Zhang, X. Meng, and T. Zhang, "Global dynamics of a virus dynamical model with cell-to-cell transmission and cure rate," Computational and Mathematical Methods in Medicine, vol. 2015, Article ID 758362, 8 pages, 2015.

[30] T. Zhang, X. Meng, and T. Zhang, "Global analysis for a delayed SIV model with direct and environmental transmissions," The Journal of Applied Analysis and Computation, vol. 6, no. 2, pp. 479-491, 2016.

[31] K. Hattaf, N. Yousfi, and A. Tridane, "Mathematical analysis of a virus dynamics model with general incidence rate and cure rate," Nonlinear Analysis: Real World Applications, vol. 13, no. 4, pp. 1866-1872, 2012.

[32] J. R. Coura and P. A. Vĩas, "Chagas disease: a new worldwide challenge," Nature, vol. 465, no. 7301, pp. S6-S7, 2010.

[33] J. A. Pérez-Molina, A. M. Perez, F. F. Norman, B. Monge-Maillo, and R. López-Vélez, "Old and new challenges in chagas disease," The Lancet Infectious Diseases, vol. 15, no. 11, pp. 1347-1356, 2015.

[34] A. L. Ribeiro, M. P. Nunes, M. M. Teixeira, and M. O. Rocha, "Diagnosis and management of Chagas disease and cardiomyopathy," Nature Reviews Cardiology, vol. 9, no. 10, pp. 576-589, 2012. 
[35] R. P. Beasley, C. Trepo, C. E. Stevens, and W. Szmuness, "The e antigen and vertical transmission of hepatitis B surface antigen," The American Journal of Epidemiology, vol. 105, no. 2, pp. 94-98, 1977.

[36] K. Hattaf and N. Yousfi, "A generalized HBV model with diffusion and two delays," Computers and Mathematics with Applications, vol. 69, no. 1, pp. 31-40, 2015.

[37] M. M. Thaler, D. W. Wara, G. Veereman-Wauters et al., "Vertical transmission of hepatitis C virus," The Lancet, vol. 338, no. 8758, pp. 17-18, 1991.

[38] X. Meng, L. Chen, and B. Wu, "A delay SIR epidemic model with pulse vaccination and incubation times," Nonlinear Analysis: Real World Applications, vol. 11, no. 1, pp. 88-98, 2010.

[39] T. Zhang, X. Meng, and T. Zhang, "SVEIRS: a new epidemic disease model with time delays and impulsive effects," Abstract and Applied Analysis, vol. 2014, Article ID 542154, 15 pages, 2014.

[40] X. Mao, Stochastic Differential Equations and Applications, Horwood Publishing, Chichester, UK, 2nd edition, 1997.

[41] E. Beretta, V. Kolmanovskii, and L. Shaikhet, "Stability of epidemic model with time delays influenced by stochastic perturbations," Mathematics and Computers in Simulation, vol. 45, no. 3-4, pp. 269-277, 1998.

[42] J. Yu, D. Jiang, and N. Shi, "Global stability of two-group SIR model with random perturbation," Journal of Mathematical Analysis and Applications, vol. 360, no. 1, pp. 235-244, 2009.

[43] N. Bacaër, "On the stochastic SIS epidemic model in a periodic environment," Journal of Mathematical Biology, vol. 71, no. 2, pp. 491-511, 2015.

[44] C. Ji and D. Jiang, "Threshold behaviour of a stochastic SIR model," Applied Mathematical Modelling, vol. 38, no. 21-22, pp. 5067-5079, 2014.

[45] T. Feng, X. Meng, L. Liu, and S. Gao, "Application of inequalities technique to dynamics analysis of a stochastic ecoepidemiology model," Journal of Inequalities and Applications, vol. 1, article 327, 2016.

[46] X. Meng, "Stability of a novel stochastic epidemic model with double epidemic hypothesis," Applied Mathematics and Computation, vol. 217, no. 2, pp. 506-515, 2010.

[47] A. Gray, D. Greenhalgh, X. Mao, and J. Pan, "The SIS epidemic model with Markovian switching," Journal of Mathematical Analysis and Applications, vol. 394, no. 2, pp. 496-516, 2012.

[48] H. C. Tuckwell and R. J. Williams, "Some properties of a simple stochastic epidemic model of SIR type," Mathematical Biosciences, vol. 208, no. 1, pp. 76-97, 2007.

[49] X. Zhang, D. Jiang, A. Alsaedi, and T. Hayat, "Stationary distribution of stochastic SIS epidemic model with vaccination under regime switching," Applied Mathematics Letters, vol. 59, pp. 87-93, 2016.

[50] Y. Cai, Y. Kang, M. Banerjee, and W. Wang, "A stochastic SIRS epidemic model with infectious force under intervention strategies," Journal of Differential Equations, vol. 259, no. 12, pp. 7463-7502, 2015.

[51] W. Zhao, J. Li, T. Zhang, X. Meng, and T. Zhang, "Persistence and ergodicity of plant disease model with Markov conversion and impulsive toxicant input," Communications in Nonlinear Science and Numerical Simulation, vol. 48, pp. 70-84, 2017.

[52] E. Tornatore, S. M. Buccellato, and P. Vetro, "Stability of a stochastic SIR system," Physica A: Statistical Mechanics and Its Applications, vol. 354, no. 1-4, pp. 111-126, 2005.

[53] A. Gray, D. Greenhalgh, L. Hu, X. Mao, and J. Pan, "A stochastic differential equation SIS epidemic model," SIAM Journal on Applied Mathematics, vol. 71, no. 3, pp. 876-902, 2011.
[54] Y. Zhao and D. Jiang, "The threshold of a stochastic SIS epidemic model with vaccination," Applied Mathematics and Computation, vol. 243, pp. 718-727, 2014.

[55] Y. Lin and D. Jiang, "Long-time behaviour of a perturbed SIR model by white noise," Discrete and Continuous Dynamical Systems. Series B, vol. 18, no. 7, pp. 1873-1887, 2013.

[56] H. Schurz and K. Tosun, "Stochastic asymptotic stability of SIR model with variable diffusion rates," Journal of Dynamics and Differential Equations, vol. 27, no. 1, pp. 69-82, 2015.

[57] Q. Lu, "Stability of SIRS system with random perturbations," Physica A. Statistical Mechanics and Its Applications, vol. 388, no. 18, pp. 3677-3686, 2009.

[58] X. Meng, S. Zhao, T. Feng, and T. Zhang, "Dynamics of a novel nonlinear stochastic SIS epidemic model with double epidemic hypothesis," Journal of Mathematical Analysis and Applications, vol. 433, no. 1, pp. 227-242, 2016.

[59] F. Wei and J. Liu, "Long-time behavior of a stochastic epidemic model with varying population size," Physica A: Statistical Mechanics and Its Applications, vol. 470, pp. 146-153, 2017.

[60] N. Dalal, D. Greenhalgh, and X. Mao, "A stochastic model of AIDS and condom use," Journal of Mathematical Analysis and Applications, vol. 325, no. 1, pp. 36-53, 2007.

[61] C. Xu, "Global threshold dynamics of a stochastic differential equation SIS model," Journal of Mathematical Analysis and Applications, vol. 447, no. 2, pp. 736-757, 2017.

[62] A. Lahrouz and A. Settati, "Necessary and sufficient condition for extinction and persistence of SIRS system with random perturbation," Applied Mathematics and Computation, vol. 233, pp. 10-19, 2014.

[63] A. Lahrouz and A. Settati, "Qualitative study of a nonlinear stochastic SIRS epidemic system," Stochastic Analysis and Applications, vol. 32, no. 6, pp. 992-1008, 2014.

[64] D. Zhao, T. Zhang, and S. Yuan, "The threshold of a stochastic SIVS epidemic model with nonlinear saturated incidence," Physica A. Statistical Mechanics and Its Applications, vol. 443, pp. 372-379, 2016.

[65] Y. Zhao, Y. Lin, D. Jiang, X. Mao, and Y. Li, "Stationary distribution of stochastic SIRS epidemic model with standard incidence," Discrete and Continuous Dynamical Systems. Series B, vol. 21, no. 7, pp. 2363-2378, 2016.

[66] Q. Liu, D. Jiang, N. Shi, T. Hayat, and A. Alsaedi, "Stationary distribution and extinction of a stochastic SIRS epidemic model with standard incidence," Physica A. Statistical Mechanics and Its Applications, vol. 469, pp. 510-517, 2017.

[67] D. Jiang, Q. Liu, N. Shi, T. Hayat, A. Alsaedi, and P. Xia, "Dynamics of a stochastic HIV-1 infection model with logistic growth," Physica A. Statistical Mechanics and Its Applications, vol. 469, pp. 706-717, 2017.

[68] Y. Cai, Y. Kang, M. Banerjee, and W. Wang, "A stochastic epidemic model incorporating media coverage," Communications in Mathematical Sciences, vol. 14, no. 4, pp. 893-910, 2016.

[69] N. T. Dieu, D. H. Nguyen, N. H. Du, and G. Yin, "Classification of asymptotic behavior in a stochastic SIR model," SIAM Journal on Applied Dynamical Systems, vol. 15, no. 2, pp. 1062-1084, 2016.

[70] N. H. Du and N. N. Nhu, "Permanence and extinction of certain stochastic SIR models perturbed by a complex type of noises," Applied Mathematics Letters, vol. 64, pp. 223-230, 2017.

[71] Q. Liu and Q. Chen, "Analysis of the deterministic and stochastic SIRS epidemic models with nonlinear incidence," Physica A. Statistical Mechanics and Its Applications, vol. 428, pp. 140-153, 2015. 
[72] Z. Chang, X. Meng, and X. Lu, "Analysis of a novel stochastic SIRS epidemic model with two different saturated incidence rates," Physica A. Statistical Mechanics and Its Applications, vol. 472, pp. 103-116, 2017.

[73] X. Zhang, D. Jiang, T. Hayat, and B. Ahmad, "Dynamics of a stochastic SIS model with double epidemic diseases driven by Lévy jumps," Physica A. Statistical Mechanics and Its Applications, vol. 471, pp. 767-777, 2017.

[74] Y. Zhou, S. Yuan, and D. Zhao, "Threshold behavior of a stochastic SIS model with Lévy jumps," Applied Mathematics and Computation, vol. 275, pp. 255-267, 2016.

[75] C. Li, Y. Pei, X. Liang, and D. Fang, "A stochastic toxoplasmosis spread model between cat and oocyst with jumps process," Communications in Mathematical Biology and Neuroscience, 2016.

[76] D. Jiang, C. Ji, N. Shi, and J. Yu, "The long time behavior of DI SIR epidemic model with stochastic perturbation," Journal of Mathematical Analysis and Applications, vol. 372, no. 1, pp. 162-180, 2010.

[77] M. Liu, C. Bai, and K. Wang, "Asymptotic stability of a twogroup stochastic SEIR model with infinite delays," Communications in Nonlinear Science and Numerical Simulation, vol. 19, no. 10, pp. 3444-3453, 2014.

[78] Q. Liu, D. Jiang, N. Shi, T. Hayat, and A. Alsaedi, "Asymptotic behavior of a stochastic delayed SEIR epidemic model with nonlinear incidence," Physica A. Statistical Mechanics and Its Applications, vol. 462, pp. 870-882, 2016.

[79] X. Li, X. Lin, and Y. Lin, "Lyapunov-type conditions and stochastic differential equations driven by G-brownian motion," Journal of Mathematical Analysis and Applications, vol. 439, no. 1, pp. 235-255, 2016.

[80] H. Ma and Y. Jia, "Stability analysis for stochastic differential equations with infinite Markovian switchings," Journal of Mathematical Analysis and Applications, vol. 435, no. 1, pp. 593-605, 2016.

[81] A. Lahrouz and L. Omari, "Extinction and stationary distribution of a stochastic SIRS epidemic model with non-linear incidence," Statistics and Probability Letters, vol. 83, no. 4, pp. 960-968, 2013. 


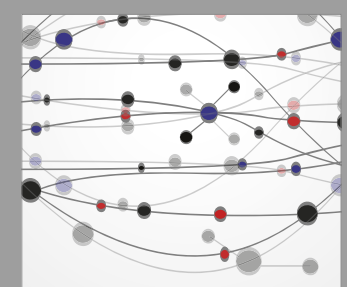

The Scientific World Journal
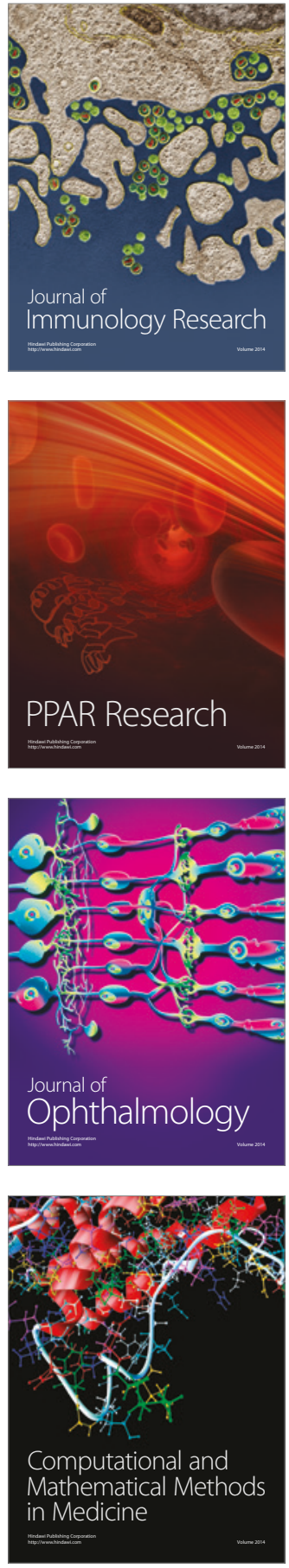

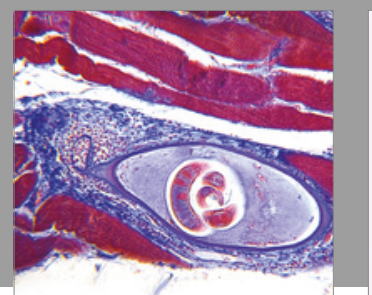

Gastroenterology Research and Practice
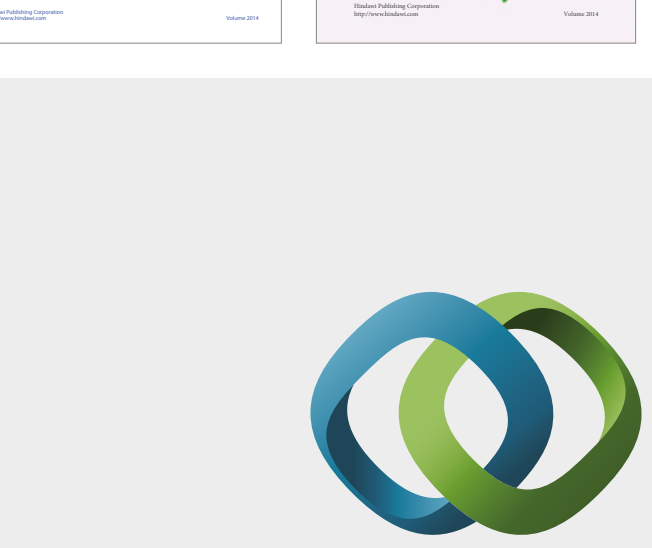

\section{Hindawi}

Submit your manuscripts at

https://www.hindawi.com
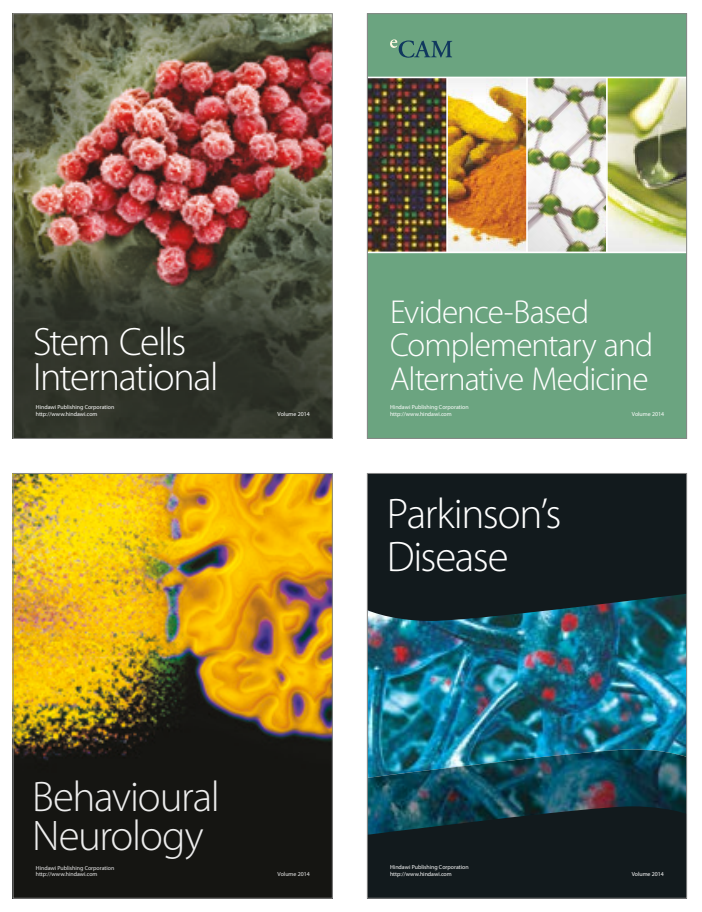
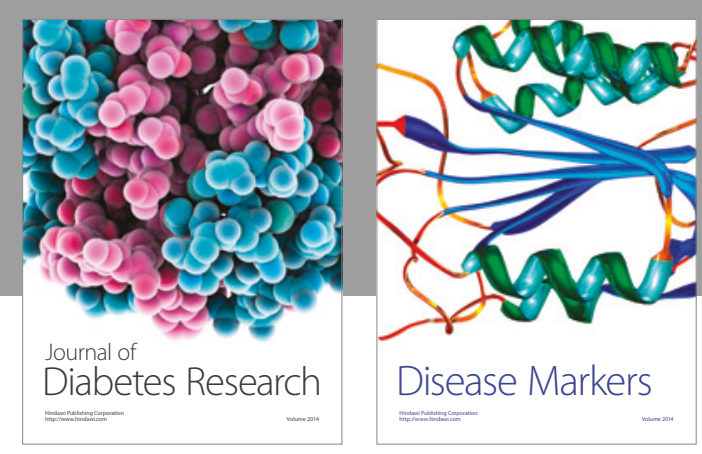

Disease Markers
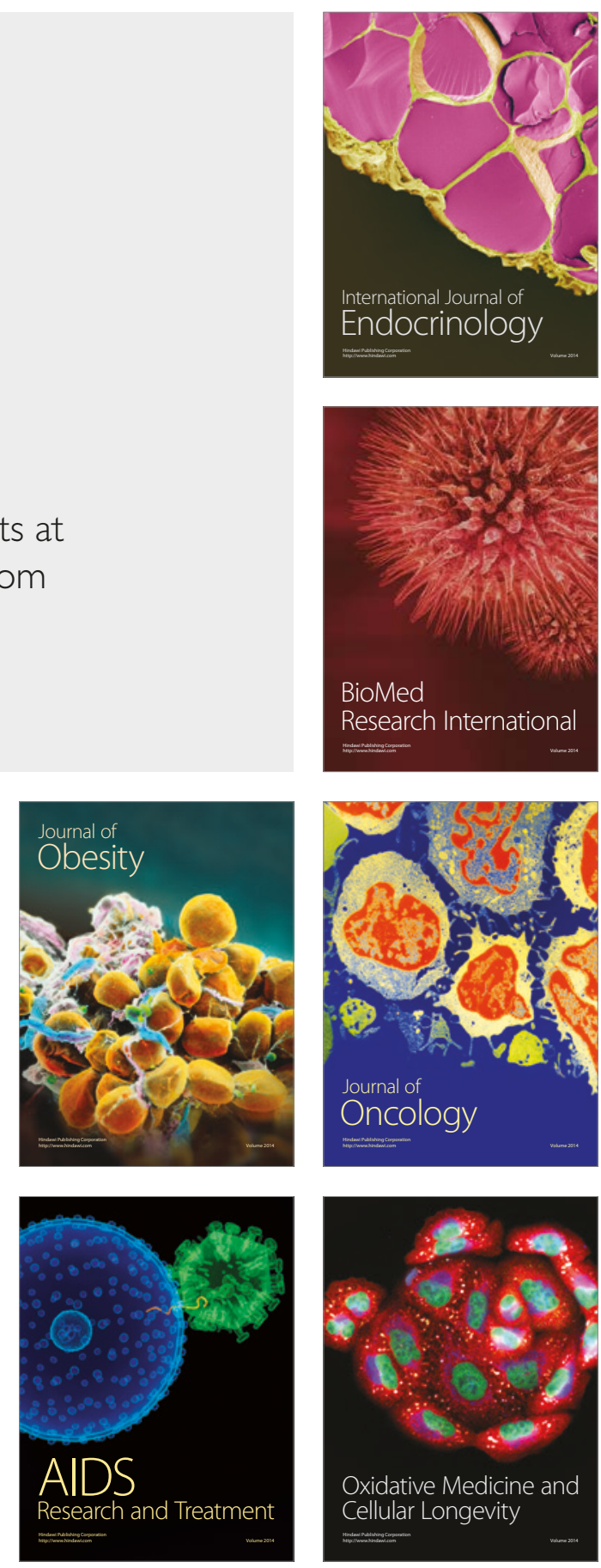